\title{
Influence of Surgical Treatment on Complications, Readmissions and Clinical Progress of Breast Cancer in Women Participating in Breast Cancer Screening Programs
}

\section{Lidia Blay ( $\square$ lydia.blay@gmail.com )}

EAHE Doctoral Program in Public Health. Department of Pediatrics, Obstetrics and Gynecology, Preventive Medicine and Public Health. Universitat Autònoma de Barcelona (UAB)

\section{Anna Jansana \\ Javier Louro \\ Joana Ferrer \\ Hospital Santa Caterina \\ Marisa Baré \\ Corporació Sanitària Parc Taulí}

Institut Hospital del Mar d'Investigacions Mèdiques

Institut Hospital del Mar d'Investigacions Mèdiques

\section{Teresa Barata}

General Directorate of Health Care Programmes, Canary Islands Health Service

\section{Laia Domingo}

Institut Hospital del Mar d'Investigacions Mèdiques

Joan Francesc Julián

Hospital Universitari Germans Trias i Pujol

\section{Xavier Castells}

Institut Hospital del Mar d'Investigacions Mèdiques

\section{Maria Sala}

Institut Hospital del Mar d'Investigacions Mèdiques

\section{Research Article}

Keywords: Mastectomy, breast conservative surgery, readmissions, complications, recurrence, mortality

Posted Date: January 18th, 2021

DOl: https://doi.org/10.21203/rs.3.rs-143351/v1

License: () (1) This work is licensed under a Creative Commons Attribution 4.0 International License. Read Full License 
Page $2 / 26$ 


\section{Abstract}

\section{Background}

Complications and readmissions derived from surgical treatment of breast cancer have been scarcely evaluated. The studies that compare mastectomy with conservative, usually focus only in recurrence and/or mortality and sometimes the results are discordant in some aspects. The aim of this study was to analyze complications and readmissions, recurrence and mortality, according to the surgical treatment received in the mammary gland.

\section{Methods}

This multicenter study included 1086 women diagnosed with breast cancer from the CaMISS cohort study of women aged between 50 and 69 years participating in 4 breast cancer screening programs in Spain between 2000 and 2009 with a follow up until 2014. Multivariate models were used to estimate the adjusted odds ratio of breast surgery (mastectomy vs conservative treatment) for complications and readmissions and hazard ratios for recurrences and mortality.

Results

Primary breast surgical treatment consisted of conservative treatment in 821 women (80.1\%) and mastectomy in 204 (19.9\%). Mastectomy was associated with readmissions, recurrences and mortality but this association was not statistically significant on multivariate adjusted analysis $(\mathrm{ORa}=1.51[95 \% \mathrm{Cl} 0.89-$ 2.57], $\mathrm{HRa}=1.37$ [95\% $\mathrm{Cl} 0.85-2.19$ ] and $\mathrm{HRa}=1.52$ [95\% $\mathrm{Cl} 0.95-2.43$ ] respectively). In our sample, the variables with greatest impact on complications, recurrences and mortality were stages III and IV $(\mathrm{ORa}=4.4[95 \% \mathrm{Cl}$ 1.22-16.16], $\mathrm{HRa}=7.96[95 \% \mathrm{Cl} 3.32-19.06]$ and $\mathrm{HRa}=3.92[95 \% \mathrm{Cl} 1.77-8.67])$.

\section{Conclusion}

Complications, readmissions, recurrence and mortality were similar in both surgical techniques. These results support that surgical treatment for breast cancer can be adapted to professional and health system circumstances, and to the surgical needs and desires of each patient.

\section{Introduction}

Breast cancer treatment is constantly changing due to ongoing advances and the need to adapt to new knowledge. It has evolved rapidly since the beginning of the 20th century, when the treatment was based solely on the extensive radical Halsted mastectomy[1]. Nowadays, international clinical practice guidelines include multiple chemotherapeutic, hormonal and immunotherapeutic treatments, as well as radiotherapy and several accepted surgical techniques[2] [3]. Depending on the characteristics of the patient (comorbidities, family history, genetic alterations, patient preferences), the tumor (tumor-node-metastases stage, histology, phenotype), and the size of the mammary gland, the patient receives the appropriate treatment following breast cancer treatment protocols and international guidelines[2] [3]. 
Survival has greatly improved due to all advances in multidisciplinary treatments[4] [5] and the early detection through screening[6] [7] [8]. However, breast cancer is still a potentially serious disease and is the most frequent form of cancer in women, with 1,671,149 new cases estimated per year worldwide, with an annual mortality of 521,907 patients[9]

Surgery plays a fundamental role in the cure of breast cancer and both mastectomy and breast conservative surgery are accepted as validated techniques. When the evidence on the effectiveness and safety of a given treatment is high, low variability in medical practice is expected[10]. However, variability in surgical practice may be a problem to face today[11] [12], with moderate-to-high variability in the performance of surgical treatment of breast cancer [10] [13] [14] [15]. After the two seminal randomized trials for breast conservation surgery, NSAPB-B06 trial[16] and Veronesi's Italian trial[17], were published, several studies have compared mastectomy with conservative surgery for the treatment of breast cancer. In some of them, no difference in mortality has been observed, although in others, the results on mortality and recurrence are discordant in some aspects. Furthermore, there are fewer studies on the impact of surgical variability on complications and readmissions. Also, studies on readmissions have focused on short-term readmissions after surgery[18] [19] [20], showing an association with postoperative complications. Only a few studies have evaluated readmissions in the long term[21] [22].

Women participating in breast cancer screening programs are more likely to be diagnosed at earlier stages and requiring less aggressive treatment modalities, having higher percentages of breast-conserving surgery and showing better survival than women diagnosed symptomatically [10] [23]. However, less attention has been paid to the impact on readmissions and treatment-related complications derived from surgical variability. A high surgical variability (mastectomy vs conservative surgery) was observed in women with the same characteristics and type of tumor in different hospitals from the CaMISS cohort study performed in Spain among women participating in breast cancer screening program[13], in agreement with other studies [10] [14] [15].

On this basis, with patients of the same age and tumor characteristics who received different surgical treatments, the aim of this current study has been to analyse complications, readmissions, recurrence and mortality according to the surgical treatment received in the mammary gland in women participating in a homogenous cohort from screening breast cancer program population.

\section{Methods}

\section{Study population}

This study included 1086 women diagnosed with breast cancer included in the CaMISS cohort. The CaMISS retrospective cohort included women aged between 50 and 69 years participating in 4 breast cancer screening programs in Spain between 2000 and 2009. This multicenter study included eight hospitals from Barcelona, Girona, Sabadell and Canary islands screening programs, and the cohort contains information on the diagnostic process, treatment, complications and follow-up until 2014[24]. 
Of the 1086 women included in the CaMISS cohort, 29 were excluded because they received only medical treatment without surgery and 32 were excluded because of missing information. The total target population included in the final analysis was 1025 patients (94.4\%) (Fig. 1).

\section{Diagnosis}

Breast cancer was detected through screening mammography or emerged as an interval cancer. In Spain, women aged between 50 and 69 years are invited to participate in a population-based screening program by a postal letter every 2 years to undergo a screening mammogram following the European guidelines for Quality Assurance in Mammographic Screening Recommendations[25]. The definition of interval cancer used was that proposed in the European guidelines as "primary breast cancer arising after a negative screening episode, with or without further assessment, and before the next screening invitation, or within 24 months for women who reached the upper age limit"[26].

The final diagnosis was obtained by biopsy of the lesion detected through an imaging test and successive histopathological study in all cases. After diagnosis, each woman was treated at the referral hospital of their screening program.

\section{Variables and data sources}

Information on patient age and detection method (screening or interval) was obtained from the databases of the population and hospital-based screening programs. Information on tumor characteristics (TNM, histology, phenotype) and treatment was obtained from medical records and from hospital-based cancer registries.

Treatment was decided in a multidisciplinary committee in each hospital. Surgical treatment was classified in two categories: conservative treatment or mastectomy. In conservative surgery, the tumor is excised with a concentric margin of healthy tissue with negative excision margins and preservation of the rest of the breast gland. Almost all patients who undergo conservative surgery receive radiotherapy. Regarding mastectomy, it consists of complete surgical resection of the breast tissue and includes all the non-conservative surgeries: modified radical mastectomy, simple mastectomy, skin-sparing mastectomy, and nipple-areolar sparing mastectomy. Mastectomy is indicated for patients who are not candidates for breast-conserving therapy, for rescue after conservative surgery that does not meet the criteria for new conservative surgery or by patient preference.

ALND was included for a better understanding of the surgical approach in each woman and because of its known relationship with complications and cancer recurrences[27]. In axillary surgery, the performance of the sentinel node biopsy (SNB) in N0 or the performance of axillary lymph node dissection (ALND) in N1/N2/N3 was recorded.

Outcome variables consisted of complications after surgical treatment, hospital readmissions, cancer recurrences and mortality. All these data were obtained from clinical records cataloged on specific annual time points from hospital-based cancer registries since the date of surgery until the end of follow-up in June 2014. 
Complications included were systemic complications, surgery-related (seroma, wound infection), pain[28] and psychological events (anxiety and/or depression).

Readmissions have been considered a complication and a loss of quality of life for patients and they were included from the surgical intervention until the end of the follow-up. The causes of readmission included surgical causes (surgery due to margin enlargement, the need to perform an ALND or mastectomy), complications of the surgical site requiring admission (infection, abscess drainage, sepsis) and complications related to systemic causes (disease progression, decompensation of concomitant diseases).

Cancer recurrence was classified in three sections: local recurrence when there was a reappearance of cancer in the ipsilateral breast, regional recurrence when the tumor involved the ipsilateral regional lymph nodes (axillar or clavicular) and metastatic when the recurrence was remote. All-cause mortality was also included.

Data were collected through a protocol approved by the clinical research ethics committee of Parc de Salut Mar (Barcelona), and the rest of the participating institutions.

\section{Statistical analysis}

A descriptive analysis including all the study variables was performed. Women's and tumor characteristics were compared by surgical treatment (conservative surgery or mastectomy) through the chi-squared test as all the study variables were categorical.

Multivariate logistic regression models were used to estimate crude and adjusted odds ratios (OR) according to complications and readmissions. Cox models were used for cancer recurrence and mortality after surgical treatment to take into account the time between the treatment and these outcomes.

The adjusted analysis included the following variables: age, diagnostic method, screening program, TNM stage, histology, phenotype, surgical treatment and performance of ALND.

Statistical significance was set at $p<0.05$. Statistical analyses were performed through the SPSS statistical package (version 23.0).

\section{Results}

Descriptive analysis of the CaMISS cohort according to the received surgical treatment

The results of the descriptive analysis are shown in Table 1. Primary breast surgical treatment included conservative treatment in 821 women $(80.1 \%)$ and mastectomy in $204(19.9 \%)$. The mean age of the patients at the time of diagnosis was $58.5 \pm 5.45$ years (49-69) with no statistically significant differences according to surgical treatment. Breast cancer was detected through screening mammograms in 713 women $(69.6 \%)$. The percentage breast cancer detected by screening mammography that underwent conservative surgery was higher than that among women with interval cancer $(73.9$ and $52 \%$, respectively, $p$ $<0.001)$. 
Conservative treatment was more frequent in stage I tumors than mastectomy $(51.4 \%$ vs $15.7 \%, p<0.001)$. Mastectomy was more frequent in women with advanced stage tumors (stage III: $19.6 \%$ vs. $6.3 \%, p<0.001$ ). The percentages of $B$ luminal and HER2 were higher in the mastectomy group than in the conservative surgery group (luminal B: $19.6 \%$ vs $23 \%$, HER2: $4.8 \%$ vs $12.3 \%, p=0.002$ ).

ALND was more frequent in the mastectomy group than in the conservative surgery group ( $78.4 \%$ vs. $58.9 \%$, $p<0.001)$. There were differences in the surgical treatment received according to the screening program $(p<$ $0.001)[13]$. 
Table 1

CAMISS cohort descriptive analysis according to the surgical treatment received

Treatment

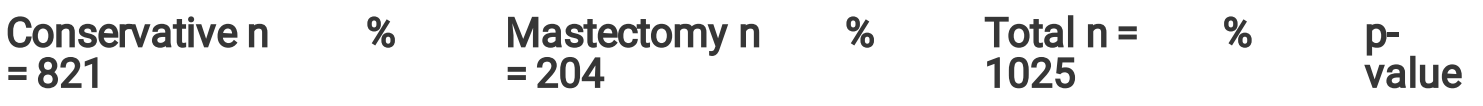

\section{Age (years)}

Median (IQR)

$57(49-69)$

$58(50-69)$

$58(49-69)$

\section{Age groups (years)}

$50-54$

$55-59$

$60-64$

65-69

Diagnostic method

Screening

Interval

TNM

In situ

I

॥

III

IV

\section{Histology}

Invasive ductal

carcinoma

Ductal carcinoma in $\quad 77$

situ

Invasive lobular

carcinoma

Others

63

72

607

$73.9 \quad 106$

26.198

$28.0 \quad 65$

$26.7 \quad 57$

$27.8 \quad 43$

$17.5 \quad 39$

$26.1-98$

$10.4 \quad 17$

$51.4 \quad 32$

$30.5 \quad 76$

$6.3 \quad 70$

$0.2 \quad 5$

605

$73.7 \quad 138$

$64.6 \quad 743$

$32.2 \quad 295$

$28.8 \quad 0.27$

$27.9 \quad 276$

26.9

$21.1 \quad 271$

26.4

$19.1 \quad 183$

17.9

value 


\section{Treatment}

$\begin{array}{llllll}\begin{array}{l}\text { Conservative } n \\ =821\end{array} & \% & \begin{array}{l}\text { Mastectomy } n \\ =204\end{array} & \% & \begin{array}{l}\text { Total } n= \\ 1025\end{array}\end{array} \quad \% \quad \begin{aligned} & \text { p- } \\ & \text { value }\end{aligned}$

\begin{tabular}{|c|c|c|c|c|c|c|c|}
\hline HER2 & 39 & 4.8 & 25 & 12.3 & 64 & 6.2 & \\
\hline Triple negative & 66 & 8.0 & 17 & 6.9 & 83 & 8.1 & \\
\hline \multicolumn{8}{|l|}{ ALND } \\
\hline Yes & 484 & 58.9 & 160 & 78.4 & 644 & 62.8 & \multirow{2}{*}{ ¿. 001} \\
\hline No & 337 & 41.1 & 44 & 21.6 & 381 & 37.2 & \\
\hline \multicolumn{8}{|l|}{ Program } \\
\hline 1 & 303 & 36.9 & 84 & 41.2 & 387 & 37.8 & \multirow{4}{*}{$\begin{array}{l}<.001 \\
0.001\end{array}$} \\
\hline 2 & 254 & 30.9 & 38 & 18.6 & 292 & 28.5 & \\
\hline 3 & 86 & 10.5 & 17 & 8.3 & 103 & 10.0 & \\
\hline 4 & 178 & 21.7 & 65 & 31.9 & 243 & 23.7 & \\
\hline
\end{tabular}

ALND: Axillary lymph node dissection

Descriptive analysis of complications, readmissions, recurrence and mortality

Complications, readmissions, recurrences and mortality outcomes by surgical treatment are shown in Table 2. A total of 292 women (28.3\%) experienced at least one complication with no differences according to surgical treatment ( $p$ value 0,744 ).

A total of 223 women (21.8\%) were readmitted after surgical treatment until the end of the follow-up. Readmissions were slightly more frequent in the mastectomy group than in the conservative treatment group $(27 \%$ and $20.5 \%$, respectively, $p=0.04)$. They predominated during the first year after surgery in patients undergoing conservative surgery $(75.6 \%)$ but were more evenly distributed during follow-up in patients undergoing mastectomy $(54.4 \%$ during the first year) $(p=0.004)$.

Regarding cancer recurrence, it affected 146 women (14.2\%) and was more frequent in the mastectomy group than in the conservative surgery group $(27.9 \%$ vs. $10.8 \%, p<0.001)$. The most frequent type of recurrence was the local one in conservative treatment (3.8\%).

The mortality rate for this cohort with a follow-up period of 13 years was $13.8 \%(n=141)$, and was higher in the mastectomy group than in the conservative surgery group ( $26.5 \%$ vs. $10.6 \%, p<0.001)$. 
Table 2

Descriptive analysis of complications, readmissions, recurrence and mortality

Treatment

\begin{tabular}{|c|c|c|c|c|c|}
\hline Conservative & $\%$ & Mastectomy & $\%$ & Total & $\%$ \\
\hline$n=821$ & & $\mathrm{n}=\mathbf{2 0 4}$ & & $\begin{array}{l}n= \\
1025\end{array}$ & \\
\hline
\end{tabular}

\section{Complications}

\begin{tabular}{llllllll} 
No & 589 & 71.7 & 144 & 70.6 & 733 & 71.5 & 0.744 \\
\cline { 1 - 7 } Yes & 232 & 28.3 & 60 & 29.4 & 292 & 28.5 &
\end{tabular}

\section{Type of}

complication

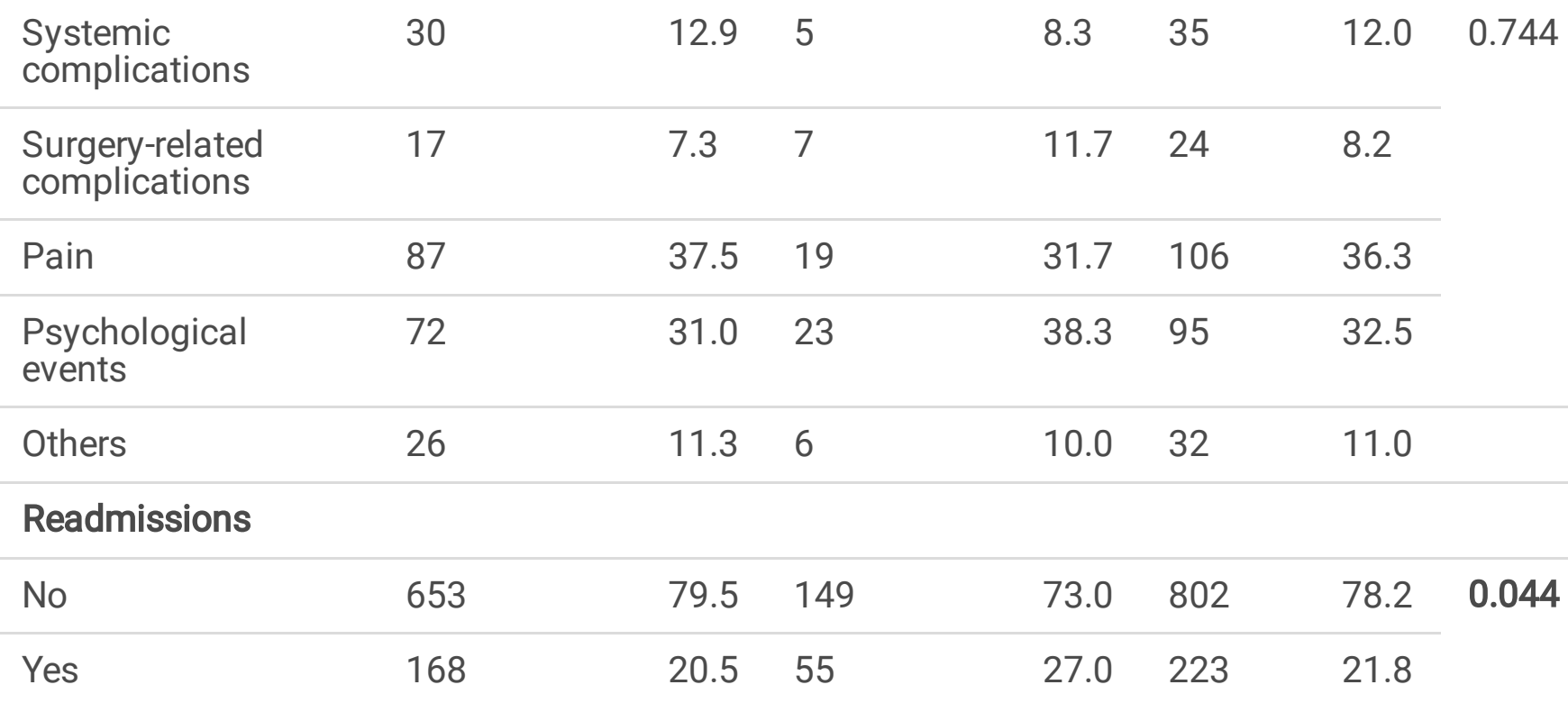

\section{Time of}

readmission

$\begin{array}{llllllll}\begin{array}{l}\leq 1 \text { year after } \\ \text { treatment }\end{array} & 127 & 75.6 & 30 & 54.5 & 157 & 70.4 & 0.004 \\ \begin{array}{l}\text { > year after } \\ \text { treatment }\end{array} & 41 & 24.4 & 25 & 45.5 & 66 & 29.6 & \end{array}$

\section{Recurrences}

\begin{tabular}{llllllll} 
No & 732 & 89.2 & 147 & 72.1 & 879 & 85.8 & $<0.001$ \\
\cline { 1 - 6 } Yes & 89 & 10.8 & 57 & 27.9 & 146 & 14.2 &
\end{tabular}

Type of recurrence

\begin{tabular}{llllllll} 
Local & 31 & 3.8 & 7 & 3.4 & 38 & 3.7 & 0.023 \\
\cline { 1 - 7 } Regional & 9 & 1.1 & 9 & 4.4 & 18 & 1.6 &
\end{tabular}




\begin{tabular}{|c|c|c|c|c|c|c|c|}
\hline & Trea & & & & & & \\
\hline $\begin{array}{l}\text { Metastatic or } \\
\text { remote }\end{array}$ & 49 & 6 & 41 & 20 & 90 & 8.9 & \\
\hline \multicolumn{8}{|l|}{ Mortality } \\
\hline No & 734 & 89.4 & 150 & 73.5 & 884 & 86.2 & $<0.001$ \\
\hline Yes & 87 & 10.6 & 54 & 26.5 & 141 & 13.8 & \\
\hline
\end{tabular}

Univariate and multivariate adjusted analysis of complications, readmissions, recurrence and mortality

In the unadjusted and adjusted OR analysis (Table 3), surgical treatment was not associated with complications $(\mathrm{ORa}=0.71[95 \% \mathrm{Cl} 0.40-1.32])$ nor with readmissions in the adjusted analysis $(\mathrm{ORa}=1.51$ [95\% $\mathrm{Cl} 0.89-2.57])$. The presence of ALND and stage III and IV tumors were associated with the presence of complications (OR a (ALND) $=3.3[95 \% \mathrm{Cl} 2.0-5.4]$ and OR a $(\mathrm{TNM})=4.4[95 \% \mathrm{Cl} 1.22-16.16])$ but not with readmissions. 
Table 3

Unadjusted and adjusted OR of complications and readmissions after surgical treatment (logistic regression)

\begin{tabular}{|c|c|c|c|c|c|c|c|c|}
\hline & \multicolumn{4}{|c|}{ Complications } & \multicolumn{4}{|c|}{ Readmissions } \\
\hline & $\begin{array}{l}\text { Unadjusted } \\
\text { OR }\end{array}$ & $\begin{array}{l}95 \% \\
\mathrm{Cl}\end{array}$ & $\begin{array}{l}\text { Adjusted } \\
\text { OR }\end{array}$ & $\begin{array}{l}95 \% \\
\mathrm{Cl}\end{array}$ & $\begin{array}{l}\text { Unadjusted } \\
\text { OR }\end{array}$ & $\begin{array}{l}95 \% \\
\mathrm{Cl}\end{array}$ & $\begin{array}{l}\text { Adjusted } \\
\text { OR }\end{array}$ & $\begin{array}{l}95 \% \\
\mathrm{Cl}\end{array}$ \\
\hline \multicolumn{9}{|l|}{ Treatment } \\
\hline $\begin{array}{l}\text { Conservative } \\
\text { surgery }\end{array}$ & 1 & 1 & 1 & 1 & 1 & 1 & 1 & 1 \\
\hline Mastectomy & 1.37 & $\begin{array}{l}0.91- \\
1.99\end{array}$ & 0.71 & $\begin{array}{l}0.40- \\
1.32\end{array}$ & 1.44 & $\begin{array}{l}1.01- \\
2.04\end{array}$ & 1.51 & $\begin{array}{l}0.89- \\
2.57\end{array}$ \\
\hline \multicolumn{9}{|l|}{ ALND } \\
\hline No & 1 & 1 & 1 & 1 & 1 & 1 & 1 & 1 \\
\hline Yes & 1.20 & $\begin{array}{l}0.89- \\
1.69\end{array}$ & 3.3 & $\begin{array}{l}2.0- \\
5.4\end{array}$ & 1.19 & $\begin{array}{l}0.79- \\
1.79\end{array}$ & 1.4 & $\begin{array}{l}0.86- \\
2.24\end{array}$ \\
\hline \multicolumn{9}{|c|}{ Age groups (years) } \\
\hline $50-54$ & 1 & 1 & 1 & 1 & 1 & 1 & 1 & 1 \\
\hline $55-59$ & 1.03 & $\begin{array}{l}0.70- \\
1.50\end{array}$ & 1.17 & $\begin{array}{l}0.67- \\
2.02\end{array}$ & 0.92 & $\begin{array}{l}0.63- \\
1.34\end{array}$ & 0.94 & $\begin{array}{l}0.57- \\
1.56\end{array}$ \\
\hline $60-64$ & 0.78 & $\begin{array}{l}0.53- \\
1.16\end{array}$ & 0.72 & $\begin{array}{l}0.40- \\
1.31\end{array}$ & 0.73 & $\begin{array}{l}0.49- \\
1.07\end{array}$ & 0.51 & $\begin{array}{l}0.29- \\
0.91\end{array}$ \\
\hline $65-70$ & 0.67 & $\begin{array}{l}0.43- \\
1.05\end{array}$ & 0.77 & $\begin{array}{l}0.40- \\
1.46\end{array}$ & 0.35 & $\begin{array}{l}0.35- \\
0.88\end{array}$ & 0.57 & $\begin{array}{l}0.30- \\
1.05\end{array}$ \\
\hline \multicolumn{9}{|c|}{ Diagnostic method } \\
\hline Screening & 1 & 1 & 1 & 1 & 1 & 1 & 1 & 1 \\
\hline Interval & 1.13 & $\begin{array}{l}0.82- \\
1.56\end{array}$ & 1.50 & $\begin{array}{l}0.94- \\
2.39\end{array}$ & 1.17 & $\begin{array}{l}0.86- \\
1.59\end{array}$ & 1.00 & $\begin{array}{l}0.09- \\
0.30\end{array}$ \\
\hline \multicolumn{9}{|l|}{ TNM } \\
\hline In situ & 0.19 & $\begin{array}{l}0.08- \\
0.45\end{array}$ & 0.35 & $\begin{array}{l}0.09- \\
1.26\end{array}$ & 0.85 & $\begin{array}{l}0.48- \\
1.48\end{array}$ & 1.04 & $\begin{array}{l}0.47- \\
2.32\end{array}$ \\
\hline । & 1 & 1 & 1 & 1 & 1 & 1 & 1 & 1 \\
\hline II & 1.67 & $\begin{array}{l}1.15- \\
2.40\end{array}$ & 1.19 & $\begin{array}{l}0.69- \\
2.06\end{array}$ & 1.41 & $\begin{array}{l}0.85- \\
2.35\end{array}$ & 1.15 & $\begin{array}{l}0.68- \\
1.96\end{array}$ \\
\hline III + IV & 1.88 & $\begin{array}{l}1.02- \\
3.47\end{array}$ & 4.44 & $\begin{array}{l}1.22- \\
16.16\end{array}$ & 1.47 & $\begin{array}{l}0.83- \\
2.61\end{array}$ & 1.07 & $\begin{array}{l}0.26- \\
4.40\end{array}$ \\
\hline
\end{tabular}

Adjusted by: treatment, ALND, age, TNM, phenotype, histology, diagnostic method and program. ALND: Axillary lymph node dissection. 


\begin{tabular}{|c|c|c|c|c|c|c|c|c|}
\hline & \multicolumn{4}{|c|}{ Complications } & \multicolumn{4}{|c|}{ Readmissions } \\
\hline & $\begin{array}{l}\text { Unadjusted } \\
\text { OR }\end{array}$ & $\begin{array}{l}95 \% \\
\mathrm{Cl}\end{array}$ & $\begin{array}{l}\text { Adjusted } \\
\text { OR }\end{array}$ & $\begin{array}{l}95 \% \\
\mathrm{Cl}\end{array}$ & $\begin{array}{l}\text { Unadjusted } \\
\text { OR }\end{array}$ & $\begin{array}{l}95 \% \\
\mathrm{Cl}\end{array}$ & $\begin{array}{l}\text { Adjusted } \\
\text { OR }\end{array}$ & $\begin{array}{l}95 \% \\
\mathrm{Cl}\end{array}$ \\
\hline \multicolumn{9}{|l|}{ Histology } \\
\hline $\begin{array}{l}\text { Invasive } \\
\text { ductal } \\
\text { carcinoma }\end{array}$ & 1 & 1 & 1 & 1 & 1 & 1 & 1 & 1 \\
\hline $\begin{array}{l}\text { Ductal } \\
\text { carcinoma in } \\
\text { situ }\end{array}$ & 0.13 & $\begin{array}{l}0.05- \\
0.33\end{array}$ & 0.11 & $\begin{array}{l}0.01- \\
2.12\end{array}$ & 0.59 & $\begin{array}{l}0.33- \\
1.07\end{array}$ & 0.11 & $\begin{array}{l}0.01- \\
1.00\end{array}$ \\
\hline $\begin{array}{l}\text { Invasive } \\
\text { lobular } \\
\text { carcinoma }\end{array}$ & 1.01 & $\begin{array}{l}0.61- \\
1.67\end{array}$ & 1.31 & $\begin{array}{l}0.63- \\
2.74\end{array}$ & 0.55 & $\begin{array}{l}0.61- \\
0.99\end{array}$ & 0.55 & $\begin{array}{l}0.24- \\
1.28\end{array}$ \\
\hline Others & 0.80 & $\begin{array}{l}0.49- \\
1.33\end{array}$ & 0.84 & $\begin{array}{l}0.36- \\
1.93\end{array}$ & 1.49 & $\begin{array}{l}0.92- \\
2.39\end{array}$ & 1.12 & $\begin{array}{l}0.52- \\
2.43\end{array}$ \\
\hline \multicolumn{9}{|l|}{ Phenotype } \\
\hline Luminal A & 1 & 1 & 1 & 1 & 1 & 1 & 1 & 1 \\
\hline Luminal B & 0.99 & $\begin{array}{l}0.65- \\
1.53\end{array}$ & 1.03 & $\begin{array}{l}0.62- \\
1.71\end{array}$ & 1.45 & $\begin{array}{l}0.93- \\
2.27\end{array}$ & 0.73 & $\begin{array}{l}0.45- \\
1.20\end{array}$ \\
\hline HER2 & 0.88 & $\begin{array}{l}0.43- \\
1.79\end{array}$ & 0.92 & $\begin{array}{l}0.40- \\
2.10\end{array}$ & 1.89 & $\begin{array}{l}0.97- \\
3.67\end{array}$ & 1.17 & $\begin{array}{l}0.55- \\
2.36\end{array}$ \\
\hline $\begin{array}{l}\text { Triple } \\
\text { negative }\end{array}$ & 1.4 & $\begin{array}{l}0.74- \\
2.63\end{array}$ & 1.13 & $\begin{array}{l}0.54- \\
2.33\end{array}$ & 1.45 & $\begin{array}{l}0.77- \\
2.76\end{array}$ & 0.87 & $\begin{array}{l}0.44- \\
1.73\end{array}$ \\
\hline
\end{tabular}

The HR analysis (Table 4) showed that mastectomy was associated with recurrences and mortality in the unadjusted analysis but the association did not remain significant after adjustment $(\mathrm{HRa}=1.37[95 \% \mathrm{Cl}$ $0.85-2.19]$ and $\mathrm{HRa}=1.52$ [95\% $\mathrm{Cl} 0.95-2.43]$ ) respectively). Among the other variables, only stage III/IV and HER2 phenotype had a statistically significant association with recurrences and mortality in the adjusted analysis, with the highest $\mathrm{HRa}$ found for stages III and IV and the risk of recurrences $(\mathrm{HRa}=7.96[95 \% \mathrm{Cl}$ 3.32-19.06]). 
Table 4

Unadjusted and adjusted HR of recurrences and mortality (COX)

\begin{tabular}{|c|c|c|c|c|c|c|c|c|}
\hline & \multicolumn{4}{|c|}{ Recurrences } & \multicolumn{4}{|l|}{ Mortality } \\
\hline & $\begin{array}{l}\text { Unadjusted } \\
\text { HR }\end{array}$ & $\begin{array}{l}95 \% \\
\mathrm{Cl}\end{array}$ & $\begin{array}{l}\text { Adjusted } \\
\text { HR }\end{array}$ & $\begin{array}{l}95 \% \\
\mathrm{Cl}\end{array}$ & $\begin{array}{l}\text { Unadjusted } \\
\text { HR }\end{array}$ & $\begin{array}{l}95 \% \\
\mathrm{Cl}\end{array}$ & $\begin{array}{l}\text { Adjusted } \\
\text { HR }\end{array}$ & $\begin{array}{l}95 \% \\
\mathrm{Cl}\end{array}$ \\
\hline \multicolumn{9}{|l|}{ Treatment } \\
\hline $\begin{array}{l}\text { Conservative } \\
\text { surgery }\end{array}$ & 1 & 1 & 1 & 1 & 1 & 1 & 1 & 1 \\
\hline Mastectomy & 2.86 & $\begin{array}{l}2.05- \\
3.99\end{array}$ & 1.37 & $\begin{array}{l}0.85- \\
2.19\end{array}$ & 2.73 & $\begin{array}{l}1.94- \\
3.83\end{array}$ & 1.52 & $\begin{array}{l}0.95- \\
2.43\end{array}$ \\
\hline \multicolumn{9}{|l|}{ ALND } \\
\hline No & 1 & 1 & 1 & 1 & 1 & 1 & 1 & 1 \\
\hline Yes & 1.20 & $\begin{array}{l}0.82- \\
1.74\end{array}$ & 0.92 & $\begin{array}{l}0.51- \\
1.65\end{array}$ & 0.99 & $\begin{array}{l}0.69- \\
1.43\end{array}$ & 0.76 & $\begin{array}{l}0.45- \\
1.35\end{array}$ \\
\hline \multicolumn{9}{|c|}{ Age groups (years) } \\
\hline $50-54$ & 1 & 1 & 1 & 1 & 1 & 1 & 1 & 1 \\
\hline $55-59$ & 0.69 & $\begin{array}{l}0.46- \\
1.04\end{array}$ & 0.56 & $\begin{array}{l}0.33- \\
0.93\end{array}$ & 0.67 & $\begin{array}{l}0.43- \\
1.07\end{array}$ & 0.62 & $\begin{array}{l}0.36- \\
1.08\end{array}$ \\
\hline $60-64$ & 0.81 & $\begin{array}{l}0.54- \\
1.20\end{array}$ & 0.71 & $\begin{array}{l}0.41- \\
1.22\end{array}$ & 0.94 & $\begin{array}{l}0.62- \\
1.43\end{array}$ & 0.86 & $\begin{array}{l}0.48- \\
1.52\end{array}$ \\
\hline $65-70$ & 0.56 & $\begin{array}{l}0.34- \\
0.93\end{array}$ & 0.49 & $\begin{array}{l}0.26- \\
0.92\end{array}$ & 1.33 & $\begin{array}{l}0.87- \\
2.04\end{array}$ & 1.37 & $\begin{array}{l}0.78- \\
2.35\end{array}$ \\
\hline \multicolumn{9}{|c|}{ Diagnostic method } \\
\hline Screening & 1 & 1 & 1 & 1 & 1 & 1 & 1 & 1 \\
\hline Interval & 1.91 & $\begin{array}{l}1.39- \\
2.61\end{array}$ & 0.98 & $\begin{array}{l}0.63- \\
1.53\end{array}$ & 2.18 & $\begin{array}{l}0.59- \\
2.98\end{array}$ & 1.33 & $\begin{array}{l}0.85- \\
2.07\end{array}$ \\
\hline \multicolumn{9}{|l|}{ TNM } \\
\hline In situ & 1.10 & $\begin{array}{l}0.49- \\
2.27\end{array}$ & 0.88 & $\begin{array}{l}0.32- \\
2.42\end{array}$ & 0.19 & $\begin{array}{l}0.05- \\
0.77\end{array}$ & 0.28 & $\begin{array}{l}0.07- \\
1.20\end{array}$ \\
\hline I & 1 & 1 & 1 & 1 & 1 & 1 & 1 & 1 \\
\hline II & 1.28 & $\begin{array}{l}0.77- \\
2.15\end{array}$ & 1.27 & $\begin{array}{l}0.65- \\
2.48\end{array}$ & 0.81 & $\begin{array}{l}0.50- \\
1.34\end{array}$ & 0.79 & $\begin{array}{l}0.43- \\
1.45\end{array}$ \\
\hline III + IV & 5.04 & $\begin{array}{l}3.01- \\
8.44\end{array}$ & 7.96 & $\begin{array}{l}3.32- \\
19.06\end{array}$ & 4.07 & $\begin{array}{l}2.26- \\
7.35\end{array}$ & 3.92 & $\begin{array}{l}1.77- \\
8.67\end{array}$ \\
\hline
\end{tabular}

Adjusted by: treatment, ANLD, age, TNM, histology, phenotype, diagnostic method, program and diagnosis date. ALND: Axillary lymph node dissection 


\begin{tabular}{|c|c|c|c|c|c|c|c|c|}
\hline & \multicolumn{4}{|c|}{ Recurrences } & \multicolumn{4}{|l|}{ Mortality } \\
\hline & $\begin{array}{l}\text { Unadjusted } \\
\text { HR }\end{array}$ & $\begin{array}{l}95 \% \\
\mathrm{Cl}\end{array}$ & $\begin{array}{l}\text { Adjusted } \\
\mathrm{HR}\end{array}$ & $\begin{array}{l}95 \% \\
\mathrm{Cl}\end{array}$ & $\begin{array}{l}\text { Unadjusted } \\
\text { HR }\end{array}$ & $\begin{array}{l}95 \% \\
\mathrm{Cl}\end{array}$ & $\begin{array}{l}\text { Adjusted } \\
\mathrm{HR}\end{array}$ & $\begin{array}{l}95 \% \\
\mathrm{Cl}\end{array}$ \\
\hline \multicolumn{9}{|l|}{ Histology } \\
\hline $\begin{array}{l}\text { Invasive } \\
\text { ductal } \\
\text { carcinoma }\end{array}$ & 1 & 1 & 1 & 1 & 1 & 1 & 1 & 1 \\
\hline $\begin{array}{l}\text { Ductal } \\
\text { carcinoma } \\
\text { in situ }\end{array}$ & 0.47 & $\begin{array}{l}0.22- \\
1.01\end{array}$ & 0.02 & $\begin{array}{l}0.00- \\
0.28\end{array}$ & 0.14 & $\begin{array}{l}0.03- \\
0.55\end{array}$ & - & - \\
\hline $\begin{array}{l}\text { Invasive } \\
\text { lobular } \\
\text { carcinoma }\end{array}$ & 1.07 & $\begin{array}{l}0.65- \\
1.75\end{array}$ & 0.97 & $\begin{array}{l}0.37- \\
2.57\end{array}$ & 0.88 & $\begin{array}{l}0.52- \\
1.51\end{array}$ & 1.32 & $\begin{array}{l}0.60- \\
2.92\end{array}$ \\
\hline Others & 0.71 & $\begin{array}{l}0.37- \\
1.35\end{array}$ & 0.26 & $\begin{array}{l}0.06- \\
1.11\end{array}$ & 1.07 & $\begin{array}{l}0.62- \\
1.83\end{array}$ & 1.04 & $\begin{array}{l}0.44- \\
3.47\end{array}$ \\
\hline \multicolumn{9}{|l|}{ Phenotype } \\
\hline Luminal A & 1 & 1 & 1 & 1 & 1 & 1 & 1 & 1 \\
\hline Luminal B & 1.38 & $\begin{array}{l}0.88- \\
2.17\end{array}$ & 1.33 & $\begin{array}{l}0.80- \\
2.20\end{array}$ & 1.10 & $\begin{array}{l}0.70- \\
1.74\end{array}$ & 1.27 & $\begin{array}{l}0.77- \\
2.09\end{array}$ \\
\hline HER2 & 3.62 & $\begin{array}{l}2.17- \\
6.03\end{array}$ & 3.39 & $\begin{array}{l}1.92- \\
5.98\end{array}$ & 2.83 & $\begin{array}{l}1.70- \\
4.70\end{array}$ & 3.01 & $\begin{array}{l}1.68- \\
5.38\end{array}$ \\
\hline $\begin{array}{l}\text { Triple } \\
\text { negative }\end{array}$ & 2.05 & $\begin{array}{l}1.18- \\
3.53\end{array}$ & 1.63 & $\begin{array}{l}0.86- \\
3.10\end{array}$ & 1.95 & $\begin{array}{l}1.15- \\
3.30\end{array}$ & 1.60 & $\begin{array}{l}0.85- \\
2.07\end{array}$ \\
\hline
\end{tabular}

\section{Discussion}

In our study population of women participating in breast cancer screening programs diagnosed with breast cancer, $80.1 \%$ patients received breast-conservative surgery and $19.9 \%$ underwent mastectomy. Mastectomy presented more readmissions, recurrences and mortality. However, after adjusting for possible confounders there was no statistically significant difference on the overall risk of complications, readmissions, recurrence and mortality between performing a conservative surgery or a mastectomy. TNM (stages III-IV) and phenotype were the factors that had a greater impact on recurrence and mortality and TNM and ALND on complications.

\section{Complications}

In our study, no differences were found in the frequency of complications depending on the type of surgical treatment. Our results showed that $28,5 \%$ of women presented complications after surgical treatment, being 
pain and psychological events the most frequent ones. This results are in line with other studies [30-34] that have shown that up to $50 \%$ of women diagnosed with breast cancer suffer from pain.

We also found that seroma was present in $8.2 \%$ of women. Seroma is a common complication after breast cancer surgery [29] [30], occurring at rates ranging from 3-85\%[31] [32] although breast surgery is categorized as a low-morbidity procedure[33] and has been described as a clean operation[34]. Some studies have reported that seromas are more likely to occur after mastectomy than after breast conservation[35] [29], while others have reported a higher rate of infection in patients undergoing mastectomy[34] [35].

As expected, statistically significant differences were found in the stage-adjusted analysis: Stages III and IV had a strong relationship with the presence of complications in adjusted analysis and the performance of an ALND involved a higher risk of complications, probably because seroma and wound infection are increased when the surgery involved lymph node dissection[34] [36]. This finding is particularly of interest in an era where the tendency is to avoid unnecessary ALND, with new protocols following the publications of $\mathrm{Dr}$ Guliano et al[37], However, in our cohort including all stages and all histologic/phenotypic kind of tumors, mortality and recurrences were equivalent between N0 patients who received SNB versus N1/N2/N3 patients who underwent ANLD.

\section{Readmisions}

Readmissions after surgical treatment occurred in $21.8 \%$ of women, being slightly higher in the mastectomy group (27\%) than in the conservative treatment group (20.5\%) but without finding statistically significant differences. Similar percentages were reported by a study performed in the United States where breastconservative surgery had a readmission rate due to reoperation of $21.6 \%$ [18]. In previous study from the same population[38] readmissions risk was not increased by surgical aproche but rather by the complications themselves. The percentage of readmissions is an official quality and hospital safety indicator in USA, Europe and other countries [39] [40] [41] and to understand and to avoid causes of readmissions is an important strategy for improving health outcomes and controlling healthcare costs[40]. In addition, admission to hospital is undoubtedly one of the most disabling situations a patient can face, both in the physical and psychological spheres[42]

\section{Recurrences}

Previous reports indicate that about $10 \%$ of women have a recurrence in the first 5 years after surgical treatment[43] [44] [45]. In our study, with a longer follow-up until 13 years (mean of 8.5 years), breast cancer recurrence affected $14.2 \%$ of women. In this study, we included women with all stages of the disease and women in stages III and IV had the highest risk of recurrences, as expected and consistent with the evidence[46] [44] [45] [47], along with patients with HER2-positive tumors[47] [48]. Women included in our study were diagnosed between 2000 and 2006 and treatment for HER2 was introduced in 2004-2005, which could explain this result, as observed in other studies where women not receiving chemotherapy or trastuzumab for HER2-postitive tumors had a significantly higher risk of recurrence[48]. As it was observed in another study[46], recurrence was more frequent in patients who underwent a mastectomy, but after the 
adjusted analysis it lose the statistical significance, probably due to the tumor characteristics and higher stages associated to this surgical technique.

The percentage of local recurrences was similar in the two surgical techniques but was slightly higher in conservative surgery (3.7\%) than in mastectomy (3.4\%). Although some studies show much higher local recurrence after conservative surgery than after mastectomy[49] [50] [51], our data agree with those in the literature, with percentages of local recurrence in patients undergoing conservative surgery of $2 \%-3.5 \%$ at 10 years, due to improvements in imaging techniques, attention to surgical margins and advances in systemic therapy[46] [52], with recurrence being equivalent to that in patients with mastectomy[53] [46].

For regional recurrences (local and nodes), our results showed a higher percentage of recurrences in women with mastectomy $(7.8 \%)$ than in those with conservative surgery $(4.8 \%)$. This result is in agreement with studies reporting regional recurrence of around $5 \%-10 \%[46]$ after surgery and with this type of recurrence being similar for the two techniques[54] [45].

Metastatic recurrences were clearly higher in women with mastectomy (20\%), as observed in another study[55]. However, a meta-analysis of nine articles on different types of mastectomies[56] found a percentage of distant recurrence after mastectomy of around $10 \%-12 \%$, even other studies described a similar metastatic recurrence in patients undergoing mastectomy and conservative surgery[50] [54].

\section{Mortality}

No difference in the adjusted risk of mortality was observed according surgical procedure.

The mortality rate in this cohort with a follow-up of up to 13 years was $13.8 \%$, which is within the range described in other studies[44] [57]. Mortality was higher in the group of women receiving mastectomy, which are the group with a less favorable prognosis, a fact that has been widely described in previous studies[58] [59] [60] [61]. Evidence in the literature is inconclusive for mortality according to the surgical technique: some studies comparing mastectomy and conservative surgery plus radiotherapy have reported similar results in terms of survival[49] [53] [50] [54] [62], even in patients with tumors $>5 \mathrm{~cm}$ [63] [64]. However, other studies suggested better survival among women treated with conservative surgery compared with mastectomy[5] [58] [59] [60] [61] [65]. In contrast, a meta-analysis published in 2016[66] indicated that mastectomy might provide a slightly overall survival benefit compared with breast-conservative therapy in early breast cancer patients with larger tumor size.

As with the most recurrent phenotypes, mortality is higher in HER2-positive and triple negative tumors, but only the statistical significance for HER2-positive tumors was maintained in the adjusted model. This study was conducted at a time when the use of monoclonal antibodies was not standardised. A recent study[67] has shown that early-stage tumors (T1N0) have the same risk of mortality regardless of phenotypic subtype, including HER2-positivity. Having estrogen and progesterone receptor-negative tumors plays a stronger role in survival than HER2 positivity for tumors of all sizes[67].

\section{Limitations And Strength}


This study has some limitations. It is based in a cohort of women participating in a population breast cancer screening program, and consequently all participants were aged between 50 and 70 years at diagnosis. This hampers comparisons with studies including women of all ages, but also lends homogeneity to the sample. The women were diagnosed between 2000 and 2009 and since then, treatment improvements have been introduced, the most important being the introduction of sentinel node biopsy and treatment for HER2positive tumors[68] [69]. Moreover, information on complications and readmissions were obtained from the medical history, which might have introduced information bias, either due to some misinformation or to variability in the quality of the information in the clinical records in the distinct hospitals. However, the clinical records review was done by trained professionals, following a common protocol, and the final models were adjusted by different screening programs. This study did not evaluate either monetary costs or patients' quality of life after surgical treatment, which are also important factors for decision making. However, few studies have analysed complications, readmissions, recurrence and mortality in the same multicenter work, with a fairly long follow-up period, taking into account tumor characteristics (including all TNM stages), diagnostic method and type of treatment in more than 1000 patients.

\section{Conclusion}

Surgeons are concerned about surgical variability in breast cancer and its related long-term outcomes. To contribute to make decisions about surgical treatment, taking into account long-term effects, it is important to have complete information about all possible outcomes.

This study supports current evidence that the results of different surgical treatment are similar: as long as safe oncological surgery is performed, breast-conserving surgery and mastectomy are equally effective in terms of complications, readmissions, recurrence and mortality adjusted by individual tumor and patients characteristics. This finding allows freer adaptation to professional and health system circumstances, and the needs and desires of each patient with the certainty that personalized surgery will not influence the prognosis of the disease, allowing us to focus on life quality of the patients.

\section{Abbreviations}

$a H R$

Adjusted Hazard ratio

$A L N D$

axillary lymph node dissection

aOR

Adjusted odds ratio

$\mathrm{Cl}$

Confidence intervals

ER

Estrogen receptor

$H R$

Hazard ratio 
Her2

Human epidermal growth factor receptor 2

OR

Odds ratio

$P R$

Progesterone receptor

SNB

sentinel node biopsy

TNM

Tumor-node-metastasis

\section{Declarations}

\section{Ethics approval and consent to participate}

Study data were collected using a protocol approved by the ethics committee of Parc de Salut Mar (CEICParc de Salut MAR), Barcelona. Specific patient consent was not required because we used retrospective data from screening participants who had previously signed information release documents. All methods were performed in accordance with the relevant guidelines and regulations.

\section{Consent for publication}

Not applicable.

\section{Availability of data and materials}

The datasets analysed during the current study are not publicly available due to privacy regulations, but are available from the corresponding author on reasonable request

\section{Competing interest}

The authors declare that they have no conflict of interest.

\section{Funding}

This work was supported by the following Grants from Instituto de Salud Carlos III, FEDER (PI16/00244) and REDISSEC, FEDER (PI16/0001/0013) The funders had no role in study design, data collection and analysis, decision to publish, or preparation of the manuscript.

\section{Authors' contribution}

LB, XC and MS designed the study. LB, AJ and MS wrote the manuscript. AJ and JL performed the statistical analyses and LB, AJ and MS contributed to the analyses and interpreted the data. JB, TB, MB, and LD were involved in data acquisition. JJ, XC and MS review and edit the manuscript and all authors read and approved the final manuscript. 


\section{Acknowledgments}

We thank Dr Pau Moreno and Dr David Parés from Germans Trias' Hospital for their suggestions and contributions to this article, as well as Mr Arnau Sans for his support.

\section{References}

[1] C. S. Bland, "The Halsted mastectomy: present illness and past history.," West. J. Med., vol. 134, no. 6, pp. 549-55, Jun. 1981.

[2] W. J. Gradishar et al., “NCCN Guidelines Insights: Breast Cancer, Version 1.2017.,” J. Natl. Compr. Canc. Netw., vol. 15, no. 4, pp. 433-451, 2017.

[3] M. B. Amin et al., "The Eighth Edition AJCC Cancer Staging Manual: Continuing to build a bridge from a population-based to a more 'personalized' approach to cancer staging," CA. Cancer J. Clin., vol. 67, no. 2, pp. 93-99, Mar. 2017.

[4] M. Kalager, M. Zelen, F. Langmark, and H.-O. Adami, “Effect of Screening Mammography on BreastCancer Mortality in Norway," N. Engl. J. Med., vol. 363, no. 13, pp. 1203-1210, Sep. 2010.

[5] S. Saadatmand, R. Bretveld, S. Siesling, and M. M. A. Tilanus-Linthorst, "Influence of tumour stage at breast cancer detection on survival in modern times: population based study in 173,797 patients.," BMJ, vol. 351, p. h4901, Oct. 2015.

[6] Independent UK Panel on Breast Cancer Screening, "The benefits and harms of breast cancer screening: an independent review," Lancet, vol. 380, no. 9855, pp. 1778-1786, Nov. 2012.

[7] Y. Gocgun et al., "Cost-effectiveness of breast cancer screening policies using simulation," The Breast, vol. 24, no. 4, pp. 440-448, Aug. 2015.

[8] M. G. Marmot et al., "The benefits and harms of breast cancer screening: an independent review.", Br. J. Cancer, vol. 108, no. 11, pp. 2205-40, Jun. 2013.

[9] M. Ghoncheh, Z. Pournamdar, and H. Salehiniya, "Incidence and Mortality and Epidemiology of Breast Cancer in the World.," Asian Pac. J. Cancer Prev., vol. 17, no. S3, pp. 43-6, 2016.

[10] M. Ridao-López, S. García-Armesto, B. Abadía-Taira, S. Peiró-Moreno, and E. Bernal-Delgado, “Income level and regional policies, underlying factors associated with unwarranted variations in conservative breast cancer surgery in Spain," BMC Cancer, vol. 11, no. 1, p. 145, Dec. 2011.

[11] M. Pera, "Variabilidad en la práctica quirúrgica. Un problema por resolver," Cirugía Española, vol. 95, no. 2, pp. 59-61, Feb. 2017.

[12] E. Rococo, C. Mazouni, Z. Or, V. Mobillion, M. Koon Sun Pat, and J. Bonastre, "Variation in rates of breast cancer surgery: A national analysis based on French Hospital Episode Statistics," Eur. J. Surg. Oncol., vol. 42, 
no. 1, pp. 51-58, Jan. 2016.

[13] L. Blay et al., "Variabilidad en la práctica de la cirugía mamaria en mujeres participantes en el programa de cribado poblacional de cáncer de mama," Cirugía Española, vol. 97, no. 2, pp. 89-96, Feb. 2019.

[14] M. A. Gilligan, R. T. Kneusel, R. G. Hoffmann, A. L. Greer, and A. B. Nattinger, “Persistent differences in sociodemographic determinants of breast conserving treatment despite overall increased adoption.," Med. Care, vol. 40, no. 3, pp. 181-9, Mar. 2002.

[15] E. Angulo-Pueyo, M. Ridao-López, N. Martínez-Lizaga, S. García-Armesto, and E. Bernal-Delgado, “Variabilidad y coste de oportunidad de las alternativas quirúrgicas en cáncer de mama," Gac. Sanit., vol. 28, no. 3, pp. 209-214, May 2014.

[16] D. L. Wickerham, S. A. Anderson, B. Fisher, R. Margolese, T. Mamounas, and N. Wolmark, “NSABP Protocol B-06: A randomized clinical trial comparing total mastectomy with lumpectomy with or without irradiation in the treatment of breast cancer - Results after 15 years of follow-up," Eur. J. Cancer, vol. 34, p. S38, Sep. 1998.

[17] U. Veronesi et al., "Twenty-Year Follow-up of a Randomized Study Comparing Breast-Conserving Surgery with Radical Mastectomy for Early Breast Cancer," N. Engl. J. Med., vol. 347, no. 16, pp. 1227-1232, Oct. 2002.

[18] J. Landercasper, E. Whitacre, A. C. Degnim, and M. Al-Hamadani, “Reasons for Re-Excision After Lumpectomy for Breast Cancer: Insight from the American Society of Breast Surgeons MasterySM Database," Ann. Surg. Oncol., vol. 21, no. 10, pp. 3185-3191, Oct. 2014.

[19] F. Gani, D. J. Lucas, Y. Kim, E. B. Schneider, and T. M. Pawlik, “Understanding variation in 30-day surgical readmission in the era of accountable care: Effect of the patient, surgeon, and surgical subspecialties," JAMA Surg., vol. 150, no. 11, pp. 1042-1049, Nov. 2015.

[20] I. Chow, P. J. Hanwright, N. M. Hansen, S. N. Leilabadi, and J. Y. S. Kim, "Predictors of 30-day readmission after mastectomy: A multi-institutional analysis of 21,271 patients," Breast Dis., vol. 35, no. 4, pp. 221-231, Nov. 2015.

[21] J. Landercasper, B. Bennie, M. S. Bray, C. A. Vang, and J. H. Linebarger, “Does neoadjuvant chemotherapy affect morbidity, mortality, reoperations, or readmissions in patients undergoing lumpectomy or mastectomy for breast cancer?," Gland Surg., vol. 6, no. 1, pp. 14-26, 2017.

[22] S. Gibson and R. McConigley, "Unplanned oncology admissions within 14 days of non-surgical discharge: a retrospective study," Support. Care Cancer, vol. 24, no. 1, pp. 311-317, Jan. 2016.

[23] P. Glasziou and N. Houssami, "The evidence base for breast cancer screening," Prev. Med. (Baltim)., vol. 53, no. 3, pp. 100-102, Sep. 2011. 
[24] "CaMISS - Cáncer de mama. Investigación en servicios sanitarios." [Online]. Available: https://www.camiss.info/. [Accessed: 17-Jul-2019].

[25] N. Perry, M. Broeders, C. de Wolf, S. Tornberg, R. Holland, and L. von Karsa, "European guidelines for quality assurance in breast cancer screening and diagnosis. Fourth edition--summary document," Ann. Oncol., vol. 19, no. 4, pp. 614-622, Oct. 2007.

[26] N. Ascunce et al., "Cancer screening in Spain," Ann. Oncol., vol. 21, no. Supplement 3, pp. iii43-iii51, May 2010 .

[27] G. H. Lyman et al., "American Society of Clinical Oncology Guideline Recommendations for Sentinel Lymph Node Biopsy in Early-Stage Breast Cancer," J. Clin. Oncol., vol. 23, no. 30, pp. 7703-7720, Oct. 2005.

[28] A. Romero et al., "Prevalence of persistent pain after breast cancer treatment by detection mode among participants in population-based screening programs," BMC Cancer, vol. 16, no. 1, p. 735, Dec. 2016.

[29] E. Hashemi, A. Kaviani, M. Najafi, M. Ebrahimi, H. Hooshmand, and A. Montazeri, "Seroma formation after surgery for breast cancer," World J. Surg. Oncol., vol. 2, no. 1, p. 44, Dec. 2004.

[30] A. J. M. van Bemmel, C. J. H. van de Velde, R. F. Schmitz, and G. J. Liefers, "Prevention of seroma formation after axillary dissection in breast cancer: A systematic review," Eur. J. Surg. Oncol., vol. 37, no. 10, pp. 829-835, Oct. 2011.

[31] E. C. Coveney, P. J. O'Dwyer, J. G. Geraghty, and N. J. O'Higgins, "Effect of closing dead space on seroma formation after mastectomy-a prospective randomized clinical trial.," Eur. J. Surg. Oncol., vol. 19, no. 2, pp. 143-6, Apr. 1993.

[32] S. Y. Boostrom et al., "Incidence of Clinically Significant Seroma after Breast and Axillary Surgery," J. Am. Coll. Surg., vol. 208, no. 1, pp. 148-150, Jan. 2009.

[33] A. F. Vitug and L. A. Newman, "Complications in Breast Surgery," Surg. Clin. North Am., vol. 87, no. 2, pp. 431-451, Apr. 2007.

[34] M. Ashraf, J. Biswas, S. Gupta, and N. Alam, "Determinants of wound infections for breast procedures: Assessment of the risk of wound infection posed by an invasive procedure for subsequent operation," Int. J. Surg., vol. 7, no. 6, pp. 543-546, 2009.

[35] P. Volkow, D. Vilar-Compte, B. Jacquemin, and C. Robles-Vidal, "Surgical Site Infections in Breast Surgery: Case-control Study,” World J. Surg., vol. 28, no. 3, pp. 242-246, Mar. 2004.

[36] C.-L. Tran, S. Langer, G. Broderick-Villa, and L. A. DiFronzo, “Does reoperation predispose to postoperative wound infection in women undergoing operation for breast cancer?," Am. Surg., vol. 69, no. 10, pp. 852-6, Oct. 2003. 
[37] A. E. Giuliano et al., "Locoregional recurrence after sentinel lymph node dissection with or without axillary dissection in patients with sentinel lymph node metastases: Long-term follow-up from the American college of surgeons oncology group (Alliance) ACOSOG Z0011 randomized trial," Ann. Surg., vol. 264, no. 3, pp. 413-419, Sep. 2016.

[38] C. Miret et al., "Factors associated with readmissions in women participating in screening programs and treated for breast cancer: A retrospective cohort study," BMC Health Serv. Res., vol. 19, no. 1, p. 940, Dec. 2019.

[39] S. R. Greysen, I. S. Cenzer, A. D. Auerbach, and K. E. Covinsky, "Functional impairment and hospital readmission in medicare seniors," JAMA Intern. Med., vol. 175, no. 4, pp. 559-565, Apr. 2015.

[40] J. Billings, J. Dixon, T. Mijanovich, and D. Wennberg, "Case finding for patients at risk of readmission to hospital: Development of algorithm to identify high risk patients," Br. Med. J., vol. 333, no. 7563, pp. 327330, Aug. 2006.

[41] D. Kansagara et al., "Risk prediction models for hospital readmission: A systematic review," JAMA Journal of the American Medical Association, vol. 306, no. 15. pp. 1688-1698, 19-0ct-2011.

[42] L. Mayhew, "On the effectiveness of care co-ordination services aimed at preventing hospital admissions and emergency attendances," Health Care Manag. Sci., vol. 12, no. 3, pp. 269-284, Jul. 2009.

[43] M. Colleoni et al., "Annual Hazard Rates of Recurrence for Breast Cancer During 24 Years of Follow-Up: Results From the International Breast Cancer Study Group Trials I to V," J. Clin. Oncol., vol. 34, no. 9, pp. 927935, Mar. 2016.

[44] A. M. Brewster et al., "Residual Risk of Breast Cancer Recurrence 5 Years After Adjuvant Therapy," JNCI J. Natl. Cancer Inst., vol. 100, no. 16, pp. 1179-1183, Aug. 2008.

[45] G. van Tienhoven et al., "Prognosis after treatment for loco-regional recurrence after mastectomy or breast conserving therapy in two randomised trials (EORTC 10801 and DBCG-82TM). EORTC Breast Cancer Cooperative Group and the Danish Breast Cancer Cooperative Group.," Eur. J. Cancer, vol. 35, no. 1, pp. 32-8, Jan. 1999.

[46] E. C. Behm et al., "Surgical margins and risk of locoregional recurrence in invasive breast cancer: An analysis of 10-year data from the Breast Cancer Treatment Quality Assurance Project," The Breast, vol. 22, no. 5, pp. 839-844, Oct. 2013.

[47] J. A. Hattangadi-Gluth et al., "Basal Subtype of Invasive Breast Cancer Is Associated With a Higher Risk of True Recurrence After Conventional Breast-Conserving Therapy," Int. J. Radiat. Oncol., vol. 82, no. 3, pp. 1185-1191, Mar. 2012.

[48] A. J. Lowery, M. R. Kell, R. W. Glynn, M. J. Kerin, and K. J. Sweeney, "Locoregional recurrence after breast cancer surgery: A systematic review by receptor phenotype," Breast Cancer Research and Treatment, vol. 133, no. 3. pp. 831-841, Jun-2012. 
[49] I. Jatoi and M. A. Proschan, "Randomized trials of breast-conserving therapy versus mastectomy for primary breast cancer: a pooled analysis of updated results.," Am. J. Clin. Oncol., vol. 28, no. 3, pp. 289-94, Jun. 2005.

[50] J. A. van Dongen et al., "Long-Term Results of a Randomized Trial Comparing Breast-Conserving Therapy With Mastectomy: European Organization for Research and Treatment of Cancer 10801 Trial," J. Natl. Cancer Inst., vol. 92, no. 14, pp. 1143-1150, Jul. 2000.

[51] G. H. de Bock, J. A. van der Hage, H. Putter, J. Bonnema, H. Bartelink, and C. J. van de Velde, "Isolated loco-regional recurrence of breast cancer is more common in young patients and following breast conserving therapy: Long-term results of European Organisation for Research and Treatment of Cancer studies," Eur. J. Cancer, vol. 42, no. 3, pp. 351-356, Feb. 2006.

[52] P. M. P. Poortmans, M. Arenas, and L. Livi, “Over-irradiation,” The Breast, vol. 31, pp. 295-302, Feb. 2017.

[53] N. Johns and J. M. Dixon, "Should patients with early breast cancer still be offered the choice of breast conserving surgery or mastectomy?," Eur. J. Surg. Oncol., vol. 42, no. 11, pp. 1636-1641, Nov. 2016.

[54] R. Arriagada, M. G. Lê, F. Rochard, and G. Contesso, "Conservative treatment versus mastectomy in early breast cancer: patterns of failure with 15 years of follow-up data. Institut Gustave-Roussy Breast Cancer Group.," J. Clin. Oncol., vol. 14, no. 5, pp. 1558-1564, May 1996.

[55] H. B. Neuman et al., "Risk of Synchronous Distant Recurrence at Time of Locoregional Recurrence in Patients With Stage II and III Breast Cancer (AFT-01)," J. Clin. Oncol., vol. 36, no. 10, pp. 975-980, Apr. 2018.

[56] S. Lanitis, P. P. Tekkis, G. Sgourakis, N. Dimopoulos, R. Al Mufti, and D. J. Hadjiminas, "Comparison of Skin-Sparing Mastectomy Versus Non-Skin-Sparing Mastectomy for Breast Cancer," Ann. Surg., vol. 251, no. 4, pp. 632-639, Apr. 2010.

[57] H. Medina-Franco et al., "Factors Associated With Local Recurrence After Skin-Sparing Mastectomy and Immediate Breast Reconstruction for Invasive Breast Cancer," Ann. Surg., vol. 235, no. 6, pp. 814-819, Jun. 2002.

[58] S. Agarwal, L. Pappas, L. Neumayer, K. Kokeny, and J. Agarwal, "Effect of Breast Conservation Therapy vs Mastectomy on Disease-Specific Survival for Early-Stage Breast Cancer," JAMA Surg., vol. 149, no. 3, p. 267, Mar. 2014.

[59] 0. J. Hartmann-Johnsen, R. Kåresen, E. Schlichting, and J. F. Nygård, "Better survival after breastconserving therapy compared to mastectomy when axillary node status is positive in early-stage breast cancer: a registry-based follow-up study of 6387 Norwegian women participating in screening, primarily operated between 1998 and 2009.," World J. Surg. Oncol., vol. 15, no. 1, p. 118, Jul. 2017.

[60] E. S. Hwang, D. Y. Lichtensztajn, S. L. Gomez, B. Fowble, and C. A. Clarke, "Survival after lumpectomy and mastectomy for early stage invasive breast cancer," Cancer, vol. 119, no. 7, pp. 1402-1411, Apr. 2013. 
[61] K. Chen, J. Liu, L. Zhu, F. Su, E. Song, and L. K. Jacobs, "Comparative effectiveness study of breastconserving surgery and mastectomy in the general population: A NCDB analysis," Oncotarget, vol. 6, no. 37, pp. 40127-40, Nov. 2015.

[62] M. Lagendijk et al., "Breast conserving therapy and mastectomy revisited: Breast cancer-specific survival and the influence of prognostic factors in 129,692 patients," Int. J. Cancer, vol. 142, no. 1, pp. 165-175, Jan. 2018.

[63] A. M. Mazor et al., "Breast conservation versus mastectomy in patients with T3 breast cancers $(>5 \mathrm{~cm})$ : an analysis of 37,268 patients from the National Cancer Database," Breast Cancer Res. Treat., vol. 173, no. 2, pp. 301-311, Jan. 2019.

[64] R. J. Bleicher et al., "Breast conservation versus mastectomy for patients with T3 primary tumors ( $>5$ cm): A review of 5685 medicare patients," Cancer, vol. 122, no. 1, pp. 42-49, Jan. 2016.

[65] S. Hofvind, Å. Holen, T. Aas, M. Roman, S. Sebuødegård, and L. A. Akslen, "Women treated with breast conserving surgery do better than those with mastectomy independent of detection mode, prognostic and predictive tumor characteristics," Eur. J. Surg. Oncol., vol. 41, no. 10, pp. 1417-1422, Oct. 2015.

[66] Y. Chen, L. Jiang, B. Gao, Z. Cheng, J. Jin, and K. Yang, "Survival and disease-free benefits with mastectomy versus breast conservation therapy for early breast cancer: a meta-analysis," Breast Cancer Res. Treat., vol. 157, no. 3, pp. 517-525, Jun. 2016.

[67] C. A. Parise and V. Caggiano, "Risk of mortality of node-negative, ER/PR/HER2 breast cancer subtypes in T1, T2, and T3 tumors," Breast Cancer Res. Treat., vol. 165, no. 3, pp. 743-750, Oct. 2017.

[68] M. J. Piccart-Gebhart et al., "Trastuzumab after Adjuvant Chemotherapy in HER2-Positive Breast Cancer," N. Engl. J. Med., vol. 353, no. 16, pp. 1659-1672, Oct. 2005.

[69] A. Schramm, N. De Gregorio, P. Widschwendter, V. Fink, and J. Huober, "Targeted Therapies in HER2Positive Breast Cancer - a Systematic Review," Breast Care, vol. 10, no. 3, pp. 173-178, Jul. 2015.

\section{Figures}




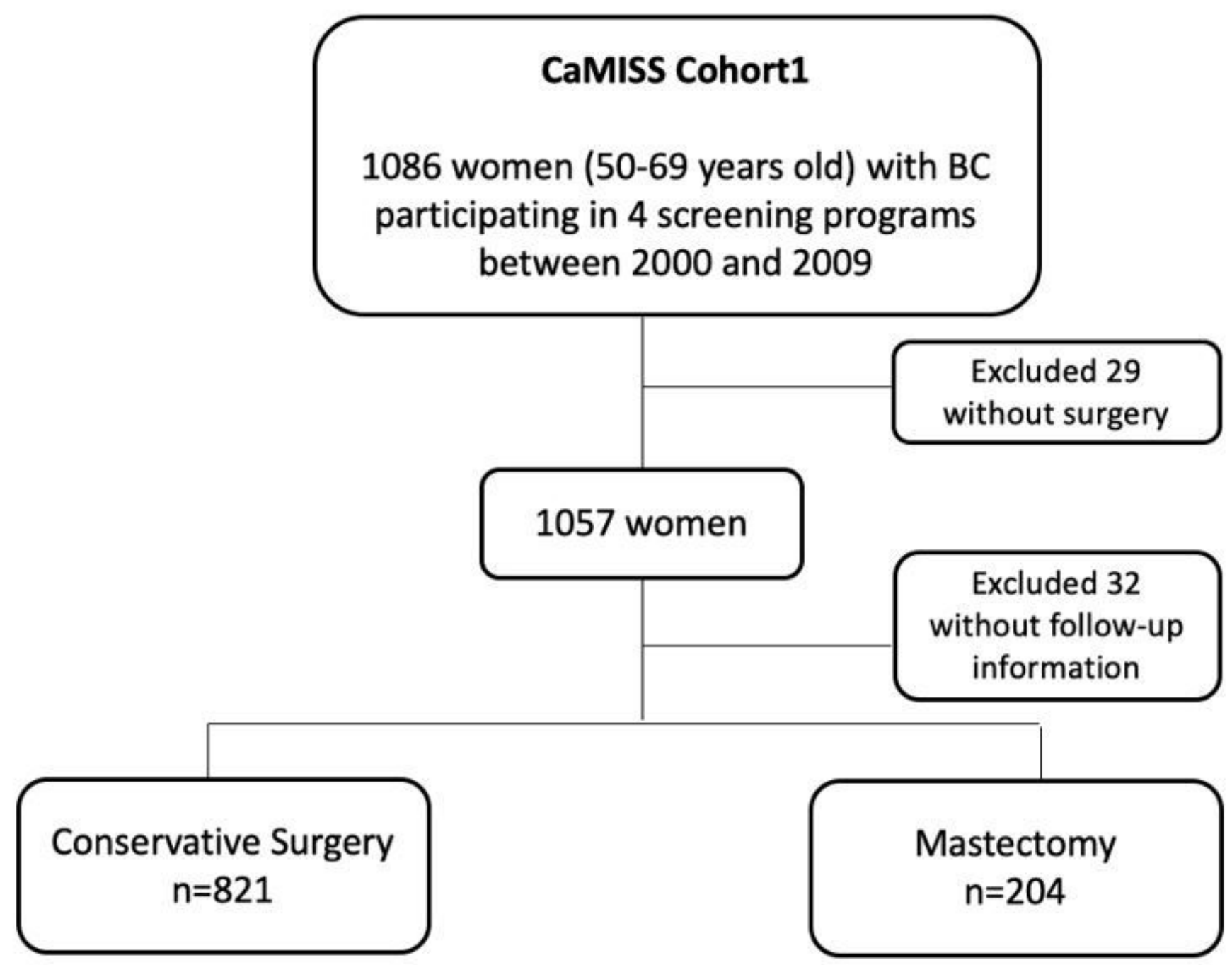

Figure 1

Study population 